\title{
Design and Development of Teaching Tools in Dimensional Geometry for Visually Impaired Students Using Object Models from 3D Printing
}

\author{
Nachaphan Junthong ${ }^{1}$, Suchapa Netpradit ${ }^{2}$ and Surapon Boonlue ${ }^{3}$ \\ ${ }^{1} \mathrm{PhD}$. Candidate of Learning Innovation and Technology Program, Faculty of Industrial Education and \\ Technology, King Mongkut's University of Technology Thonburi, Thailand \\ ${ }^{2}$ Department of Printing and Packaging Technology, King Mongkut's University of \\ Technology Thonburi, Thailand \\ ${ }^{3}$ Department of Learning Technology and Mass Communication, King Mongkut's University of Technology \\ Thonburi, Thailand
}

\begin{abstract}
This research aims to design and develop the teaching tools for blind students in the elementary level by creating the object models with $3 D$ printing. The information was collected from the interview of blind students in upper elementary and lower secondary level, and expert teachers of the blind schools in Thailand. Their opinion was concluded that mathematics subject is the most important but the most difficult to understand for the blind students, especially topic of geometry. There is much need of teaching tools to enhance the blind student's interpretation of geometry by their touch and encourage their learning to create the geometric shapes manually. The object models as teaching tool should contain the descriptive braille for the blinds and should be colorful to stimulate the attention of other low-vision students in the class. After that, all information of need was used to develop and design the teaching tools for blind students by drafting with a 3D software (SketchUp). The simulated objects in real dimension with different style of braille and relief line was then produced by $3 D$ printer using digital model data under computer control. The optimum proportion of the braille for easy reading and the relief line for easy learning were evaluated and chosen by the touch of blind children. The teaching application was also tested by the teachers of Mathematics subject in a blind school. The object models were adjusted until the style, size and thickness were optimal as the requirement. Finally, the developed models of geometry board was designed including the component parts for learning and understanding several geometric shapes either in $2 D$ or $3 D$.
\end{abstract}

Keywords: Geometry, Object Models, 3D Printing, Teaching Tools, Visually Impaired Students

\section{Introduction}

In Thailand, the blind school uses the core curriculum of basic education in 2008 [1] as well as the normal children, which differ in the special needs education because the blind are not being able to learn from the visible. According to the Education for Disabilities Act, the school has set up an environment for teaching and learning support systems; technology, facilities, services, media and educational assistance for people with disabilities to be accessible [2]. Therefore, teachers need to add appropriate equipment or learning support media for blind children to understand the lessons which cannot learn by sight. However, learning by listening alone does not help blind children to understand the context in which they are exposed. Therefore, media for blind students should be designed and developed to be effective for learning by touching. In previous work, the researcher survey the status and needs for instructional media of blind students from upper elementary school in Thailand by interviews of blind students at the elementary and junior high school level including experienced teachers teaching blind or sighted students [3]. The goal of media production is that Mathematics is the most important subject for blind students but the most difficult to understand, especially the geometry. 
Geometry and spatial sense is the best taught through concrete hands on experiences when instructing students who are blind or visually impaired. This is beneficial not only for students with visual impairments but for students with normal vision as well [4]. Generally, a geometry books for the blind are in braille without the picture as a relief in the paper, instead of a simple ink drawing. If a visually accessible workbook is not available, the teacher or another student can easily make such a thing, for example by tracing the lines of a picture with a needle. In the school for the blind, when the students have a decent intuitive grasp of what lines and other geometrical shapes look like, they should be able to draw the figures themselves. A geoboard is a mathematical manipulative used to explore basic concepts in plane geometry such as perimeter, area and the characteristics of triangles, squares and other polygons. It consists of a physical board with a certain number of nails half driven in, around which are wrapped geo bands. Learners stretch bands around the pegs to form line segments and polygons and make discoveries about perimeter, area, angles, congruence, fractions, and more. Two-dimensional geoboard may be used to help capture a student's explanations of the concept they have discovered or illustrated on the geoboard.

The objective of this research is to identify the blind student's interpretation of geometry subject in the fifth grade of elementary school. The result can inspire teachers to design further learning for development of blind student geometry concepts. The blind student can interpret the model shapes represents their mental image of three-dimensional geometry that they can't visualize directly by using traditional geoboard. In addition, the enjoyable model will enhance the learning engagement of students by increasing their attention and focus. The 3D models can motivate them to practice higher-level critical thinking skills and helps teachers more successfully achieve the course's learning objectives.

To product the needed model shape, 3D printing can be used as the process in which material is joined or solidified under computer control to create a three-dimensional object. The objects can be formed almost any shape or geometry using digital model from SketchUp software. The 3D printing deposits a binder material onto a powder bed with inkjet printer heads layer by layer for rapid prototyping. In this study, the object model of teaching tools was created by using 3D printing to enhance learning, understanding, attention and respond to self-learning of blind children as much as possible.

\section{Methodology}

\subsection{Information Survey on Visually Impaired Students}

The questionnaire included of general information, subjects with understanding problems, the need of creating media to enhance learning and the teaching media that teachers use. The semi-structured interviews were conducted with the sampling group of blind students in elementary and junior high schools level and teachers who have experienced teaching in blind schools in different parts of Thailand. The information was further used to design and develop the media to support the learning needs and enhance the visual understanding.

\subsection{Study of 3D Printing System}

The 3D printer in this study was Flashforge Creator Pro with a model dimension size of $230 \times 150 \times 150$ $\mathrm{mm}$, layer resolution of 50-300 micron and print speed of 10-200 mm/sec. The plastic for injection was PLA type which is good property to be easily printed with slight shrinkage and no odor while printing. It must be melted at $210^{\circ} \mathrm{C}$ and solidified at room temperature. After injection from the print head, the object models are

high strength and environmental friendly. The program used to run the printer is FlashPrint which can be downloaded from the owner's website and applied in a computer with 64-bit windows. 


\subsection{Information Analysis for Teaching Tool Design}

The information from the survey was analyzed to find the ideas in teaching tool design such as the key components, the suitable model and size for application and the details to be identified on the tools. The 3D models were drawn in the SketchUp program before sending the digital file to control the 3D printer. Many tool components were created as prototypes with different styles and sizes for evaluation. The suitable functions such as the braille size, the joined line height, the pin-hole depth and the normal letter size for teacher reading were chosen from the teachers of mathematics and visually impaired students in a blind school. After evaluation the appropriate tool components for reading and learning by touching, the models were then corrected as the evaluators' opinion. The optimum size, shape and thickness of the components were further designed to develop the new models of "Geometry Board" which could enhance the teaching and learning of dimensional geometry.

\subsection{Development of Geometry Board}

The optimum size and style of components from the study was used to design the new model of geometry board which was developed from the traditional one. The actual model of geometry board included of many components such as a pin-studded z-axis in the slot for learning the dimensional shape of geometry were created by plastic injection with a 3D printer. The stainless steel pins with a height of $17 \mathrm{~mm}$ and diameter $3 \mathrm{~mm}$ were also prepared to use for stretching the rubber band with a diameter of about $3 \mathrm{~cm}$. The prototype of developed geometry board to be applied as a new teaching tools for geometry learning will be further evaluated.

\section{Results and Discussion}

\subsection{Needs of Blind Students and Teachers in Teaching Tool Development}

The survey results showed that mathematics is the most important and difficult for blind students which have high demand for tool development, especially geometry. This issue matches the research of Andriyani et al. [5] that is visualization becomes one of the important ability used to help students in understanding spatial concepts, shapes, sizes and distances. However, blind students lose visual aid to learn the concept of geometry and losing of visual experience causes some difficulties in obtaining the concept of geometry directly. Their favorite learning tool is the real thing, embossed images and braille book, respectively. The important features are embossed images for touch able with braille description and 3D models. The blind children need modern technology to improve their ability as normal people, corresponding to Buteau et al. [6] that is technology can eliminate the tediousness of calculations, allowing students to focus more on conceptual understanding.

The teachers of mathematics also agreed that the teaching tools for geometry should help children drawing the geometric shape by themselves. The tools should enhance them in practice reading, writing and understanding for the real object which should be modern, suitable and portable. Approximately $95 \%$ of blind students do not understand the mathematics because there are no embossed images of geometry, graph, shapes etc. The braille codes contain too much styles to remember, read and understand. The students have to repeatedly read by self-learning and need special teachers to help. This problem is according to Vianna [7] that students with visual impairment have difficulty of geometry images understanding. The difficulty of learning geometry is not only experienced by students, but also by teachers who teach this subject. Although using tools in physical models, many teachers have difficulty to instruct geometry to blind students [8]. However, teachers suggested that the size of tool should not be larger than their hands to survey the area for learning by touching. Teachers also prefer the bright and colorful tools to motivate their normal students' attention.

\subsection{Appropriate Proportion of the Components in Teaching Tools}

The simulation of the Geometry board was used to evaluate the use of the blind students. The comparison between the grooved lines and the embossed lines in different levels are shown in Figure 1. The blind students chose the D line, which is the convex line of the $1.5 \mathrm{~mm}$ height from plane because the line is the easiest to read. The A line is a grooved line that is $1.5 \mathrm{~mm}$ depth which was difficult to find. The $\mathrm{B}$ and $\mathrm{C}$ line is raised from the 
plane of $0.5 \mathrm{~mm}$ and $1.0 \mathrm{~mm}$, respectively. However, the maximum height of raising line was the best for learning by finger-touching.

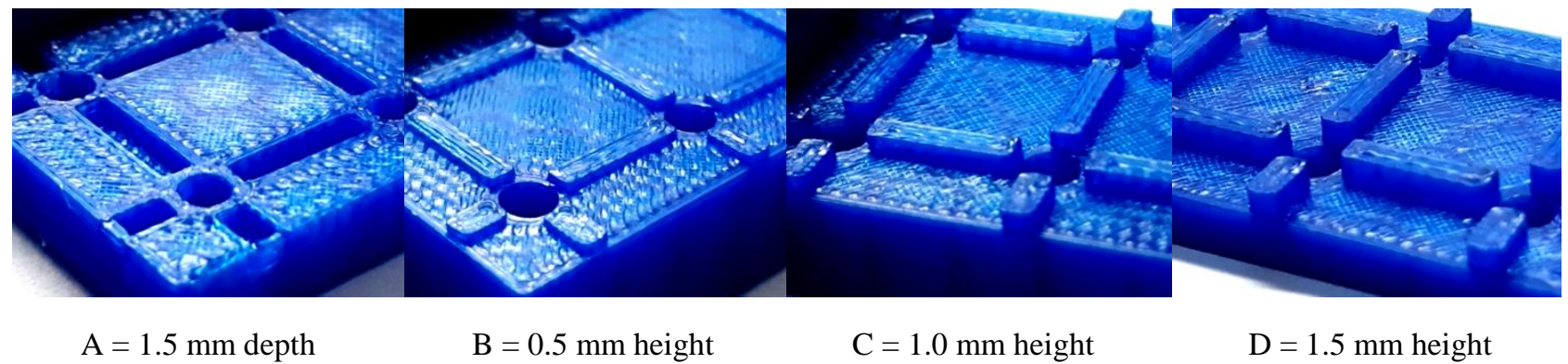

Fig.1 The different styles of joining lines: A, B, C and D for choosing by theblind students.

From the different braille sizes in Figure2, the blind students chose the Braille E size which was the largest proportion. The diameter of the braille point is $1.5 \mathrm{~mm}$, the distance between each point of the braille is $1.5 \mathrm{~mm}$ and the height of the floor is $1.5 \mathrm{~mm}$. The $\mathrm{C}$ size of the Braille font is approximately the same as the normal font used in all Braille printers but the students did not choose this size. The diameter of the braille point in $\mathrm{C}$ size is $1 \mathrm{~mm}$, the distance between each point of the braille is $1 \mathrm{~mm}$ and the height is raised from the flat surface to $1 \mathrm{~mm}$. Although the mention size was the setting size used in the drawing process, the actual size was slightly changed after modeled with 3D printer. The deviation size was also considered to adjust in the software later.

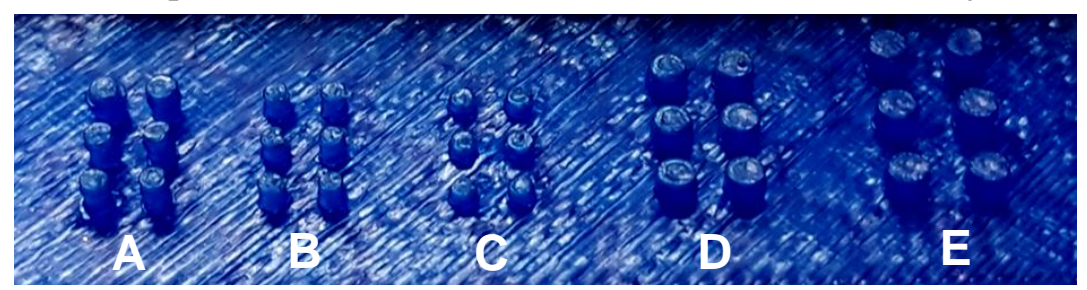

Fig. 2 The different sizes of braille characters: A, B, C, D and E for choosing by the blind students.

The evaluation with different depth of pin holes are shown in Figure 3. The appropriate level or size of models were chosen by the blind students before using to determine the design proportions of the point, Braille and pin hole of the new designed and developed geometry board.

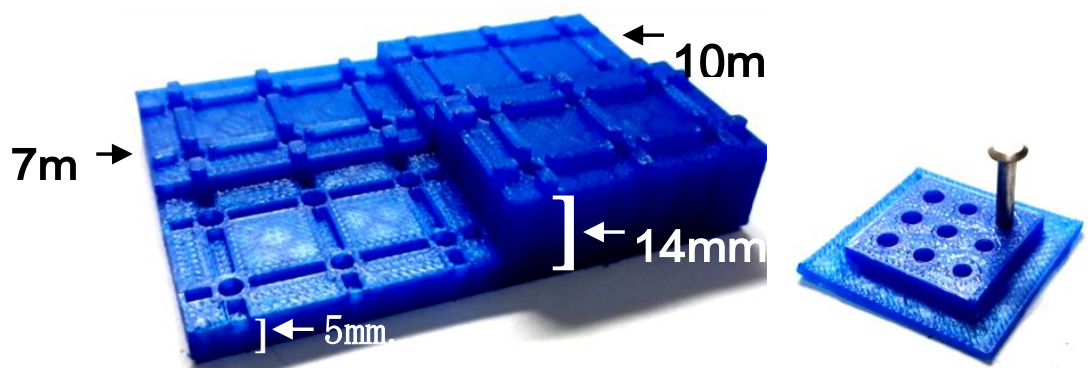

Fig. 3 The different board thickness and hole depth for choosing in geometric board design.

It was found that the design of the board thickness and the depth of the pin holes were very important, due to the strength of the pins that stretch the shrink back of rubber band. If the board is not much thicker enough, the depth of the holes will be too less, and the pins could not be fixed to the board. The depth of holes was related to the thickness of the board, the optimum depth of the pin hole was $14 \mathrm{~mm}$ and the board thickness was then made of $14 \mathrm{~mm}$ thickness which made the pin head rise above the board plane of about $3 \mathrm{~mm}$. The optimum diameter for pin hole spacing was $3.4 \mathrm{~mm}$ which was slightly larger than the pin diameter of $3 \mathrm{~mm}$. 


\subsection{Development of Teaching Tool for 3D Geometry}

The results of the evaluation is used to created the model of teaching tools which helps blind children to understand the imagination both 2D (x,y) and 3D (z-axes) by touching and can also create the geometric shapes. The picture shown the comparison of the prototype geometry board [9] and the new board was designed and developed as shown in Figure 4.
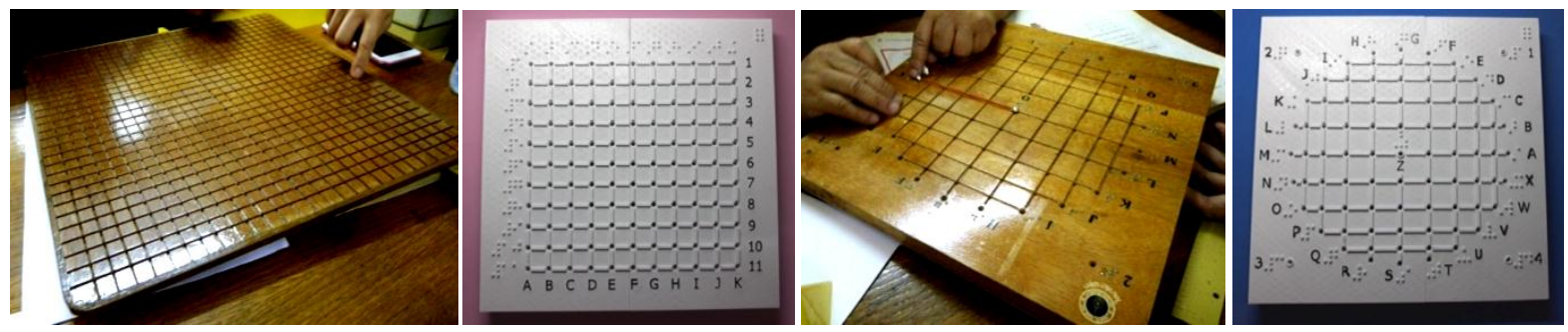

Fig. 4 The traditional wooden geometry board compared to the prototype PLA geometry board.

The designed geometry boards were developed from the traditional board as the following details.

1) The size of the new boards were reduced from the original board (left) which is $16 \times 16$ inches with 28 vertical slots and 28 horizontal slots. The size of new board was reduced to $8 \times 8$ inches with 10 vertical slots and 10 horizontal slots. The original board (right) is $12 \times 12$ inches and the diameter of the circle is 9 inches. The size of new board was reduced to $8 \times 8$ inches and the diameter of the circle is reduced to 6 inches. This reasons were considered from area of a blind student's hands for easier touching and drawing on geometry. The lighter weight of board is easier to move and better stick on the desk due to the soft texture material underneath which was also flexible, friction and colorful.

2) The joining line of point-to-point is more easily to find because the traditional wooden board used deepen groove and hole. The new developed boards use a raised line of $1.5 \mathrm{~mm}$ above the plane and have braille description for each point. Teachers can use the letters A to $\mathrm{K}$ and numbers 1 to 11 as scales to direct children to create geometry shapes. The bigger size of Braille is also used as the satisfaction of the blind children.

3) The PLA board material is durable, lightweight and colorful. The old board is brown wood which is polished castings, very heavy and too large. There are also need more accessories such as rubber bands and vertical axes to connect with. The new board are colorful and enhance the attention of visual impairment who study in the blind school to enhance mutual learning between visual impairment children and blind children.

4) Traditional geometry boardused to create geometry in $2 \mathrm{D}$ ( $\mathrm{X}, \mathrm{Y}$ axes) only, such as triangles and polygons but the new designed and developed board can create 3D geometry shapes. The pillars for creating 3D geometry shapes with vertical or $\mathrm{z}$ axes which can installed or remove as needed. Moreover, the geometry boards can create various dimensional shapes to understand the geometry of the triangle pyramid or square pyramid. This new board can support the understanding of area calculating and also volume calculating while the old board is used to understand the area only.
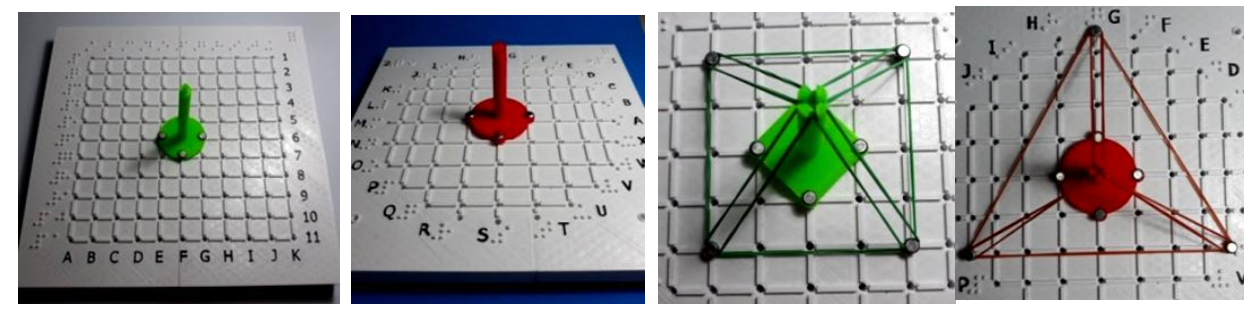

Fig. 5 The pillar of $Z$ axes which can be installed or removed as needed on a developed geometry board.

Figure 5 shows that the pillar of $\mathrm{Z}$ axes can be installed or removed as needed to understand and create the geometric shapes in both 2D and 3D on the geometry boards. The vertical axes designs were also adjusted to be 
easily placed into the slots as shown in Figure 6 which has more advantages than the first design. The part used to attach the board is designed to be a plug like a thumb tacks without complicated base on the board. However, the disadvantage is that it is easy to break when it comes to the traction of multiple strands of rubber bands that must be used together to form a geometry. Since the high tensile strength can cause tilts, this problem might be resolved by using a larger rubber bands to reduce the pulling force.

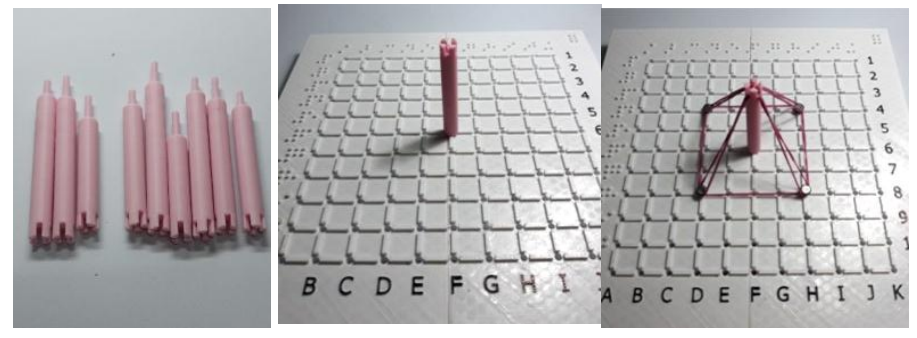

Fig. 6 The re-designed $\mathrm{Z}$ axes for easier installation or removal.

\section{Conclusion}

The appropriate teaching tool for geometry board should help the blind student to learn and understand of visualization in both 2D (x,y axes) and 3D (z-axes) by hand touching. The dimension area should not be bigger than two hands for the children to survey in a short time and not too much details because this will make the blind children confused and waste times in learning and might be misunderstanding in the learning outcome. The geometry board development media can enhance the learning skills by using the components to draw the geometry themselves. It is marked with a braille and a convex dot that defines each corner of the triangle or rectangle. Developing with a scale of units that can be used to calculate areas of geometry shapesand the volume.

In blind school, the tools should be designed and developed for visual impairment children as well. In the case of using different surface levels for blind children and colorful for visual impairment students to attract the attention for learning. The surface of the embossed on the tool model should be easily distinguish for reading by touching and the material surface should be significantly different but should not be affected to hands or skin irritation. For this reasons, the PLA plastic was used due to the safety for touching, non-toxic and environmentally friendly.

\section{References}

[1] The Ministry of Education Thailand, Basic Education Curriculum2008,Bangkok: Council Printing House,2008, pp. 7190.

[2] Government Gazette, “Enactment education Management for the disabled 2008, ”Bangkok: Council Printing House, vol. 125, ch.28, pp. 4, February 2010.

[3] N.Junthong and team, "The Study of Status and Needs for Instructional Media of Blind Students from Upper Elementary School in Thailand," presented at International Conference on Education, Humanities and Social Sciences Studies (EHSSS-(17, Singapore, March 27-28, 2017.

[4] Carmen Willings. (2018). "Teaching Students with Visual Impairments.” Retrieved February 12, 2018, from :https://www.teachingvisuallyimpaired.com/geometry--spatial-sense.html

[5] Thinus-Blanc C and Gaunet F. (1997). Representation of space in blind persons: Vision as a spatial sense?.Psychological Bulletin. [Online]. 121(1).pp. 20-42. Available: http://dx.doi.org/10.1037/0033-2909.121.1.20

[6] Buteau, C., Marshall, N., Jarvis, D., \&Lavicza, Z. (May 2010). Integrating computer algebra systems in postsecondary mathematics education: Preliminary results of a literature review. International Journal ofSpecial Education. [Online].17(2), pp. 57-68. Available: http://casresearch.nipissingu.ca/documents/ IJTME\%20(2010).pdf 
[7] Vianna C S, Barbosa P M, Rocha D F and Silva B. (2008). Teaching Geometry for Blind and Visually Impaired Students. Journal of Physics: Conference Series. [Online]. Vol. 947.Available: http://iopscience.iop.org/article/10.1088/1742-6596/947/1/012055/meta

[8] Pritchard C K and Lamb J H. (Aug 2012). Teaching Geometry to Visually Impaired Students. Journal of Mathematics Teacher. [Online]. Vol. 106, pp. 22-27.Available: https://eric.ed.gov/?id=EJ988757

[9] J. Chaiyadech, "Efficiency of using Geoboard compare with Geometric tactile materials : Case study of fifth grade students with Visual Impairment at school for the blind." M.S. thesis, M.A. (Rehabilitation Science for Persons with Disabilities), Ratchasuda Foundation (college), Mahidol Univ., Thailand. 2011.

[10]P. Pijukkana and team, "The Development of Learning Aid "Thailand Map" for Children with Visual Impairments," research, Dept. Architecture and Design. Arch.,Rajamangala Univ., Bangkok, Thailand, 2011.

[11] P. Satitvittayanand, “The Children's book design for Encouraging the Image of Blind Children," M.S. thesis, Dept. Visual Communication Design. Fine Arts.,Silpakorn Univ., Bangkok, Thailand, 2003.

[12] Andriyani, I K Budayasa and D Juniati. (2018). The blind student's interpretation of two-dimensional shapes in geometry." Journal of Physics: Conference Series. [Online]. Vol 947. Available: http://iopscience.iop.org/article/10.1088/1742-6596/947/1/012055/meta 